\title{
Table 3: Schools with Disability Studies Courses
}

(By country and alphabetically)

(Legend of codes at the end)

\begin{tabular}{|c|c|c|c|c|}
\hline \multicolumn{5}{|c|}{ Canada } \\
\hline UNIVERSITY & DISCIPLINE & $\begin{array}{l}\text { Level - \# } \\
\text { Courses }\end{array}$ & COURSE Title & $\begin{array}{l}\text { Offered } \\
\text { (last yr) }\end{array}$ \\
\hline Brock U & $\begin{array}{l}\text { Applied } \\
\text { Disability } \\
\text { Studies }\end{array}$ & G-1 & $\begin{array}{l}\text { ADST 5F00 Foundations in Applied DS and the Study of } \\
\text { Exceptionality }\end{array}$ & $\mathrm{Y}$ \\
\hline Carleton U & Social Work & UG-1 & 430 - Social Work and Persons with Disabilities & $\mathrm{Y}$ \\
\hline $\begin{array}{l}\text { King's U.C at } \\
>\text { U Western Ontario }\end{array}$ & Sociology & UG-1 & Soc 3339 f/g Social Construction of Disabilities & $\mathrm{Y}$ \\
\hline Lakehead U & Sociology & UG-1 & Sociology of Disability: Soc 4511 & $\mathrm{Y}$ \\
\hline Laurentian U & $\begin{array}{l}\text { Social Work }+ \\
\text { Con. Ed. }\end{array}$ & UG-1 & $\begin{array}{l}\text { SWLF } 3736 \text { E Critical Perspectives on Disability } \\
\text { (Distance education format) }\end{array}$ & $\mathrm{Y}$ \\
\hline McGill U & Social Work & G-1 & SWRK 669 Disability and Rehabilitation & $\mathrm{Y}$ \\
\hline Ryerson U & Liberal Studies & UG-1 & History of Madness & $\mathrm{Y}$ \\
\hline St. Francis Xavier U & Anthropology & UG-1 & Anth 236 - Disability and Society & $(2005)$ \\
\hline
\end{tabular}




\begin{tabular}{|c|c|c|c|c|}
\hline Simon Fraser U & $\begin{array}{l}\text { Women's } \\
\text { Studies }\end{array}$ & UG-1 & Special Topics: Women and Disability & $\overline{\mathrm{N}}$ \\
\hline \multirow[t]{4}{*}{ U of British Columbia } & \multirow[t]{4}{*}{$\begin{array}{l}\text { Centre for } \\
\text { Women's \& } \\
\text { Gender Studies }\end{array}$} & \multirow[t]{4}{*}{ G-4 } & $\begin{array}{l}\text { EDST 565D:Feminist Critical DS, Resilience, Representation \& } \\
\text { Justice in Education }\end{array}$ & $\mathrm{N}$ \\
\hline & & & $\begin{array}{l}\text { EDST565F:Dis/ability, Representation \& Social Justice: Disabling } \\
\text { States, Vulnerable Citizens \& Nation-Building }\end{array}$ & $\mathrm{N}$ \\
\hline & & & $\begin{array}{l}\text { EDST565A: Seminar in Disability, Social Justice, Education \& } \\
\text { Work }\end{array}$ & $\mathrm{N}$ \\
\hline & & & $\begin{array}{l}\text { EDST565A: The Medicalization of Education \& Society: In/visible } \\
\text { Citizens in The Unruly Salon Act Up }\end{array}$ & $\mathrm{Y}$ \\
\hline U of Saskatchewan & English & G-1 & $\begin{array}{l}\text { English } 818.6 \text { (01): Studies in Methods and Texts: Constructions of } \\
\text { Disability and Illness in Literature and Culture }\end{array}$ & $\mathrm{Y}$ \\
\hline \multirow[t]{6}{*}{ U of Toronto } & OSIE/SESE & G-1 & SES 1957 Doing Disability in Theory and Everyday Life & $\mathrm{Y}$ \\
\hline & \multirow[t]{5}{*}{ Equity Studies } & \multirow[t]{5}{*}{ UG-6 } & $\begin{array}{l}\text { NEW349H1F - Special Topics in DS: Disability Culture and Social } \\
\text { Change }\end{array}$ & $\mathrm{Y}$ \\
\hline & & & NEW241Y1Y - Introduction to DS & $\mathrm{Y}$ \\
\hline & & & $\begin{array}{l}\text { NEW344Y1Y - Special Topics in Equity Studies: Equity and the } \\
\text { Body }\end{array}$ & $\mathrm{Y}$ \\
\hline & & & NEW344H1F- Special Topics in DS: Disability and Representation & $\mathrm{Y}$ \\
\hline & & & $\begin{array}{l}\text { NEW449H1S - Advanced Special Topics in DS: Contemporary } \\
\text { Theories in Disability }\end{array}$ & $\mathrm{Y}$ \\
\hline
\end{tabular}




\begin{tabular}{|c|c|c|c|c|}
\hline & $\begin{array}{l}\text { Women and } \\
\text { Gender Studies }\end{array}$ & G-2 & $\begin{array}{l}\text { WGS366H1F- Special Topics in DS: Disability Culture and Social } \\
\text { Change }\end{array}$ & $\overline{(2007)}$ \\
\hline & & & WGS366H1 - Gender and Disability & $\mathrm{Y}$ \\
\hline \multirow[t]{4}{*}{ U of Victoria } & $\begin{array}{l}\text { Human and } \\
\text { Social } \\
\text { Development }\end{array}$ & UG-1 & $\begin{array}{l}\text { HSD } 464 \text { Introduction to DS } \\
\text { New course code: SOCW } 469\end{array}$ & $\begin{array}{l}(2007) \\
Y\end{array}$ \\
\hline & $\begin{array}{l}\text { Studies in Policy } \\
\text { and Practice }\end{array}$ & G-1 & SPP 530 Special Topics: Disability/Chronic Illness & $\mathrm{Y}$ \\
\hline & Sociology & UG-1 & 365 Women Disabilities/Disabilities in Society & $(2002)$ \\
\hline & $\begin{array}{l}\text { Continuing } \\
\text { Education }\end{array}$ & UG-1 & Disabilities in Society & $(2008)$ \\
\hline \multirow[t]{2}{*}{ U of Winnipeg } & Sociology & UG-2 & Introduction to DS I (50.1003/3) -3 sections & \multirow[t]{2}{*}{$\mathrm{Y}$} \\
\hline & & & Introduction to DS II (SOC.-05.1004/3) & \\
\hline
\end{tabular}




\begin{tabular}{|c|c|c|c|c|}
\hline \multicolumn{5}{|c|}{ Australia and New Zealand } \\
\hline UNIVERSITY & DISCIPLINE & $\begin{array}{l}\text { Level - \# } \\
\text { Courses }\end{array}$ & COURSE Title & $\begin{array}{l}\text { Offered } \\
\text { (last yr) }\end{array}$ \\
\hline LaTrobe U & School of Law & G-1 & LAW5RDC Human Rights and Comparative Disability Law & $\mathrm{Y}$ \\
\hline U of Newcastle & $\begin{array}{l}\text { School of } \\
\text { Education }\end{array}$ & G-1 & $\begin{array}{l}\text { EDUC6001 DS: Perspectives on Individual, Family \& Community } \\
\text { (distance ed. format) }\end{array}$ & $\mathrm{Y}$ \\
\hline U of Queensland & Social Work & UG-1 & $\begin{array}{l}\text { SWSP3076 Social Work Practice in Disability } \\
\text { (distance ed. format) }\end{array}$ & $\mathrm{Y}$ \\
\hline
\end{tabular}




\begin{tabular}{|c|c|c|c|c|}
\hline \multicolumn{5}{|c|}{ United Kingdom } \\
\hline UNIVERSITY & DISCIPLINE & $\begin{array}{l}\text { Level - \# } \\
\text { Courses }\end{array}$ & COURSE Title & $\begin{array}{l}\text { Offered } \\
\text { (last yr) }\end{array}$ \\
\hline Lancaster U. & $\begin{array}{l}\text { Applied Social } \\
\text { Science (Social } \\
\text { Work) }\end{array}$ & UG-1 & ASSC107 Disability in Society & $\mathrm{Y}$ \\
\hline U of Aberdeen & $\begin{array}{l}\text { Divinity and } \\
\text { Religious Studs }\end{array}$ & UG-1 & $\begin{array}{l}\text { DR4539 Theological Explorations in Disability, Community } \\
\text { and Humanness }\end{array}$ & $(2003)$ \\
\hline \multirow[t]{2}{*}{ U of Bath } & $\begin{array}{l}\text { Social \& Policy } \\
\text { Sciences }\end{array}$ & UG-1 & SP30098: Disability \& Society & $(2004)$ \\
\hline & Education & G-1 & ED10181 Experience of Disability & $(2007)$ \\
\hline U of Birmingham & Neuroscience & G-1 & Module1; The Needs \& Rights of People with Learning Disabilities & $\mathrm{Y}$ \\
\hline U of Exeter & Education & UG-1 & Disability (ECY3004) & $\mathrm{Y}$ \\
\hline \multirow[t]{2}{*}{ U of Glasgow } & Urban Studies & UG-1 & EX89ET Disability and Society & $(2007)$ \\
\hline & $\begin{array}{l}\text { Equality and } \\
\text { Human Rights }\end{array}$ & G-1 & The Disabling Society & $\mathrm{n} / \mathrm{s}$ \\
\hline U of Gloucestershire & Social Work & UG-1 & SW314 Disability, Impairments and Social Work & $\mathrm{Y}$ \\
\hline U of Sheffield & Education & UG-1 & EDU6054 Debates in DS & $\mathrm{N} / \mathrm{S}$ \\
\hline
\end{tabular}




\begin{tabular}{|c|c|c|c|c|}
\hline \multicolumn{5}{|c|}{ United States of America } \\
\hline UNIVERSITY & DISCIPLINE & $\begin{array}{l}\text { Level - \# } \\
\text { Courses }\end{array}$ & COURSE Title & $\begin{array}{l}\text { Offered } \\
\text { (last yr) }\end{array}$ \\
\hline Adelphi U & Sociology & UG-1 & 0952-110-11 Self \& Society: Disability in America & $\mathrm{N} / \mathrm{S}$ \\
\hline \multirow[t]{2}{*}{ Brown U } & $\begin{array}{l}\text { Community } \\
\text { Health }\end{array}$ & UG-1 & $\begin{array}{l}\text { BC168 -Pathologies to Power: Disability, Health, and } \\
\text { Community }\end{array}$ & $(2006)$ \\
\hline & German & UG-1 & German 166 - Disability in German and American Culture & $(2005)$ \\
\hline City U, NY & Theatre & UG-1 & THEA 244 Drama and Disability & $\mathrm{N} / \mathrm{S}$ \\
\hline \multirow[t]{3}{*}{ Davidson College } & \multirow[t]{3}{*}{ English } & \multirow[t]{3}{*}{ UG-4 } & ENG100 Extraordinary Bodies & \multirow[t]{3}{*}{$\mathrm{N} / \mathrm{S}$} \\
\hline & & & ENG393 Disability \& Literature & \\
\hline & & & $\begin{array}{l}\text { ENG493/4 Disability Literature/Disability \& } 20^{\text {th }} \text { Century } \\
\text { American Drama }\end{array}$ & \\
\hline \multirow[t]{2}{*}{ Emory U } & $\begin{array}{l}\text { Sociology and } \\
\text { Religion }\end{array}$ & UG-1 & SR612. DS in Religion & $\mathrm{N} / \mathrm{S}$ \\
\hline & English & G-1 & ENG717 Milton & $\mathrm{N} / \mathrm{S}$ \\
\hline Florida State U & Theatre & UG/G-1 & Disability \& Representation & $\begin{array}{l}\text { (Spring2009)* } \\
\text { every other } \\
\text { year }\end{array}$ \\
\hline Fordham U & English & UG-1 & ENLV 3843-001 - Extraordinary Bodies & $\mathrm{Y}$ \\
\hline George Washington U & $\begin{array}{l}\text { Women's } \\
\text { Studies }\end{array}$ & G-1 & WSTU 270.11 - Gender, Disability, and Film & $\mathrm{N} / \mathrm{S}$ \\
\hline Indiana U & Gender Studies & G-1 & $\begin{array}{l}\text { Graduate Topics in Gender Studies: Disability Between Law \& } \\
\text { Culture }\end{array}$ & $(2007)$ \\
\hline Indiana U of PA & Sociology & G-1 & SOC 552 Disability and Society & $\mathrm{N} / \mathrm{S}$ \\
\hline
\end{tabular}




\begin{tabular}{|c|c|c|c|c|}
\hline \multirow{2}{*}{ Lamar University } & \multirow{2}{*}{$\begin{array}{l}\text { Deaf Studies \& } \\
\text { Deaf Education }\end{array}$} & \multirow{2}{*}{ G-2 } & Intro to Deaf Studies & \multirow{2}{*}{$\mathrm{N} / \mathrm{S}$} \\
\hline & & & Intro to Deaf Education & \\
\hline Metropolitan State U & $\begin{array}{l}\text { Human Services } \\
\text { and Disability } \\
\text { Studies }\end{array}$ & UG-3 & HSDS 305 - Disability Rights: History and Current Trends & $\mathrm{N} / \mathrm{S}$ \\
\hline Northwestern U & $\begin{array}{l}\text { Education and } \\
\text { Social Policy }\end{array}$ & UG-1 & SESP Student Organized Seminar on DS & $(2005)$ \\
\hline Pacific U & English & UG-1 & ENG-221 Disability \& Literature & $\mathrm{N} / \mathrm{S}$ \\
\hline Portland State U & English & UG-1 & Eng305U Images of Disability in Lit. \& Film & $(2006)$ \\
\hline Rowan U & $\begin{array}{l}\text { Social \& } \\
\text { Behav'l } \\
\text { Sciences/ } \\
\text { Interdisciplinary }\end{array}$ & UG-1 & INTR01 168 (4901.168) - What's Wrong With Normal? & $\begin{array}{l}\text { N/S (No } \\
\text { URL) }\end{array}$ \\
\hline \multirow[t]{2}{*}{ San Fran State U } & History & \multirow[t]{2}{*}{ UG-2 } & History 642: History of The Disability Minorities in America & $(2001)$ \\
\hline & Philosophy & & Phil 382 Feminism and Disability & $\mathrm{N} / \mathrm{S}$ \\
\hline San Jose State U & $\begin{array}{l}\text { Special } \\
\text { Education }\end{array}$ & UG-1 & EDSE103 Disability \& Society & $\mathrm{N} / \mathrm{S}$ \\
\hline $\begin{array}{l}\text { SUNY } \\
>U \text { of Albany }\end{array}$ & Interdisciplinary & UG-1 & Uni 230 An Introduction to DS & $\mathrm{Y}$ \\
\hline Suffolk U & History & UG-1 & HST 367 Disability in America & $\mathrm{N} / \mathrm{S}$ \\
\hline Temple U & $\begin{array}{l}\text { Institute on } \\
\text { Disabilities }\end{array}$ & UG-1 & TR0813: Disability Identity & $\mathrm{N} / \mathrm{S}$ \\
\hline U of Alabama & English & UG-2 & HON/EH- 49201: Crip Cultures and DS & $\begin{array}{l}\text { (1999) (No } \\
\text { URL) }\end{array}$ \\
\hline
\end{tabular}




\begin{tabular}{|c|c|c|c|c|}
\hline & Sociology & & CFE500/SPE 621: Sociology of Disability & $\begin{array}{l}(1998)(\text { No } \\
\text { URL) }\end{array}$ \\
\hline \multirow[t]{5}{*}{ U of Arizona } & \multirow{3}{*}{$\begin{array}{l}\text { Special } \\
\text { Education, } \\
\text { Rehabilitation, } \\
\text { and Psychology }\end{array}$} & \multirow[t]{5}{*}{ UG-5 } & SERP 414 - Introduction to DS/Services & $\mathrm{Y}$ \\
\hline & & & SERP 399/499/599 Universal Design \& Accessible Space & $\mathrm{N} / \mathrm{S}$ \\
\hline & & & SERP 416 - Disability Perspectives and Narratives & $\mathrm{N} / \mathrm{S}$ \\
\hline & $\begin{array}{l}\text { Women's } \\
\text { Studies }\end{array}$ & & $\begin{array}{l}\text { WS } 400 / 500 \text { - The History of Reproduction: From Eugenics to } \\
\text { Reproductive Technology }\end{array}$ & $\mathrm{N} / \mathrm{S}$ \\
\hline & History & & HIST 495F - History of Disability: A Global Perspective & $\mathrm{N} / \mathrm{S}$ \\
\hline $\begin{array}{l}\text { U of California } \\
\text { >San Diego }\end{array}$ & Cultural Studies & UG-1 & LTCS 87: Disability in Film & $\mathrm{Y}$ \\
\hline \multirow{2}{*}{$\begin{array}{l}\text { U of Illinois } \\
>\text { Urbana Champaign }\end{array}$} & History & \multirow[t]{2}{*}{ UG-2 } & 498H Research and Writing Seminar, Topic: Disabilities & $(2005)$ \\
\hline & $\begin{array}{l}\text { Rehabilitation } \\
\text { Counselling }\end{array}$ & & REHB 330 Disability in American Society & $\mathrm{Y}$ \\
\hline U of Iowa & History & UG-1 & 16A:106 Disability in American History & $\mathrm{Y}$ \\
\hline U of Michigan & $\mathrm{N} / \mathrm{S}$ & G-1 & Rackham 580: Topics in DS: Disability and Health & $\mathrm{Y}$ \\
\hline $\begin{array}{l}\text { U of Minnesota } \\
>\text { Morris }\end{array}$ & $\begin{array}{l}\text { Interdisciplinary } \\
\text { Studies }\end{array}$ & UG-1 & IS1331 Disability in America: Politics, Art, Culture(s) & $(2003)$ \\
\hline $\begin{array}{l}\text { U of Missouri } \\
>\text { Kansas City }\end{array}$ & Humanities & UG-1 & $\begin{array}{l}\text { Humanities 409P: Perceptions \& Images of Disabilities in Film } \\
\text { and Literature }\end{array}$ & $\mathrm{N} / \mathrm{S}$ \\
\hline $\begin{array}{l}\text { U of Nebraska } \\
>\text { Lincoln }\end{array}$ & English & G-1 & ENGL 898 Special Topics -"Disability \& Literature & $\begin{array}{l}\text { (2002) } \\
\text { (No URL) }\end{array}$ \\
\hline
\end{tabular}




\begin{tabular}{|c|c|c|c|c|}
\hline \multirow[t]{8}{*}{ U of Oregon } & English & \multirow[t]{8}{*}{ G-8 } & ENG615: Disability Theory in the Humanities Grad Seminar & Y \\
\hline & $\begin{array}{l}\text { International } \\
\text { Studies }\end{array}$ & & INTL410/510: Global Perspectives on Disability & Y \\
\hline & \multirow{5}{*}{$\begin{array}{l}\text { Special } \\
\text { Education }\end{array}$} & & CDS201: Communication Disorders in society and Media & $\mathrm{Y}$ \\
\hline & & & $\begin{array}{l}\text { SPED410/510: Global Perspectives on Disability } \\
* \text { cross listed with INTL410/510 }\end{array}$ & $\mathrm{Y}$ \\
\hline & & & SPED411: Foundations of Disability I & $\mathrm{Y}$ \\
\hline & & & SPED412: Foundations of Disability II & $\mathrm{Y}$ \\
\hline & & & SPED430: Introduction to Exceptionalities & $\mathrm{N} / \mathrm{S}$ \\
\hline & $\begin{array}{l}\text { Family and } \\
\text { Human Services }\end{array}$ & & FHS_(?)_: Disability Imagery in Film & $\mathrm{N} / \mathrm{S}$ \\
\hline U of Pittsburgh & $\begin{array}{l}\text { Health } \\
\text { Information } \\
\text { Management }\end{array}$ & G-1 & HRS2708 The Individual \& Social Experience of Disability & $\mathrm{N} / \mathrm{S}$ \\
\hline \multirow[t]{5}{*}{$\begin{array}{l}\text { U of Texas } \\
>\text { Austin }\end{array}$} & \multirow{5}{*}{$\begin{array}{l}\text { Social Work/ } \\
\text { Texas Centre for } \\
\text { Disability } \\
\text { Studies }\end{array}$} & \multirow[t]{5}{*}{ UG-7 } & $\begin{array}{l}\text { SW 360K/SW 387R Introduction to DS: The Social Context of } \\
\text { Disability }\end{array}$ & $(2005)$ \\
\hline & & & $\begin{array}{l}\text { SW 360K/SW 387R Introduction to DS: Making Systems } \\
\text { Work for People with Disabilities }\end{array}$ & $(2006)$ \\
\hline & & & $\begin{array}{l}\text { SW 360K/SW 387R Family Support, Self-Determination, and } \\
\text { Disability }\end{array}$ & $(2006)$ \\
\hline & & & SW 360K/SW 387R Aging and Disability & $\mathrm{N} / \mathrm{S}$ \\
\hline & & & SW 360K/SW 387R Women and Disability & $\mathrm{Y}$ \\
\hline
\end{tabular}




\begin{tabular}{|c|c|c|c|c|}
\hline & & & $\begin{array}{l}\text { SW 360K/SW 387R Developmental Disabilities and Self- } \\
\text { Advocacy }\end{array}$ & $(2005)$ \\
\hline & & & $\begin{array}{l}\text { SW 360K/SW 387R Psychiatric Disabilities: From the Inside } \\
\text { Out }\end{array}$ & $(2006)$ \\
\hline \multirow[t]{3}{*}{ U of Virginia } & \multirow{3}{*}{$\begin{array}{l}\text { Arts and } \\
\text { Sciences }\end{array}$} & \multirow[t]{3}{*}{ UG-3 } & ASL481- Deafness in Literature and Film & $\mathrm{N} / \mathrm{S}$ \\
\hline & & & ASL475 - Topics in Deaf Studies & $(2006)$ \\
\hline & & & ASL230- Women and Gender in the Deaf World & $\mathrm{N} / \mathrm{S}$ \\
\hline U of Washington & Philosophy & G-1 & $\begin{array}{l}\text { 540A-Seminar in Ethics - Dependency and Disability in } \\
\text { Moral and Political Theory }\end{array}$ & $(2004)$ \\
\hline $\begin{array}{l}\text { U of Washington } \\
>\text { Bothell }\end{array}$ & $\begin{array}{l}\text { Interdisciplinary } \\
\text { Studies }\end{array}$ & UG-1 & BIS 325 Disability and Human Rights & $\mathrm{Y}$ \\
\hline Wayne State U & $\begin{array}{l}\text { Developmental } \\
\text { Disabilities } \\
\text { Institute } \\
\end{array}$ & G-1 & SW6740 - Seminar in DS & $\mathrm{N} / \mathrm{S}$ \\
\hline Western Illinois U & $\begin{array}{l}\text { English and } \\
\text { Journalism }\end{array}$ & UG-1 & GH 101: Disability and Normalcy in Literature & $(2005)$ \\
\hline Yale U & English & UG-1 & ENGL 329a, The Spectacle of Disability & $\mathrm{Y}$ \\
\hline
\end{tabular}

\section{Codes Legend:}

\begin{tabular}{ll}
\hline Con Ed & Continuing Education \\
UG & Undergraduate \\
$G$ & Graduate \\
Y & Currently offered \\
$(200 x)$ & (Last year course was offered)
\end{tabular}

